diagnosis of Attention Deficit Hyperactivity Disorder (ADHD) or other cognitive or attachment difficulties. Six families completed feedback questionnaires. Most parents felt that the process helped their understanding of their child. Follow-up support was identified as an area for improvement. The clinicians found the process helpful, particularly with patients for whom there was disparity of opinion about that child.

Conclusion This model demonstrated positive patient experience, increased diagnostic accuracy and clinicians reported improved job satisfaction. It is likely to shorten initial referral to diagnosis time and offer a model of working that is closer to NICE guidance. Cost implications would need to be considered. Should this model of working be commissioned, areas for improvement would include follow up after diagnosis, and the robustness of the administration around the clinic.

\section{G630(P) INNOVATIVE APPROACH -DEVELOPMENT OF A PATHWAY FOR FORMULARY APPROVAL FOR MEDICINES FOR THE TREATMENT OF YOUNG PEOPLE WITH ATTENTION DEFICIT HYPERACTIVITY DISORDER IN A SECONDARY CARE SETTING IN WEST MIDLANDS, UK JOINTLY BY CHIEF PHARMACIST AND PAEDIATRICIAN (SPECIALIST) IN 2017}

${ }^{1} \mathrm{R}$ Jainer, ${ }^{1,2} \mathrm{~J}$ Yates. ${ }^{1}$ Paedatrics Department, Universty hospitals of Birmingham NHS Trust, Solihull, UK; ${ }^{2}$ Pharmacy, University Hospitals of Birmingham NHS Trust, Solihull, UK

\subsection{6/archdischild-2020-rcpch.544}

Aims This innovative process was undertaken to examine the most effective route to ensure that young people consistently received evidenced-based cost-effective treatment for Attention Deficit Hyperactivity Disorder (ADHD)

Method A formulary approval pathway was developed for ADHD medication, following consultation, for a consistent approach.

A Specialist expressed interest in medication was reviewed by the formulary team, which involved a series of questions to be answered:

- Demonstrates evidence of benefit to the recipient and the health economy.

- Is there a strong evidence base for medication?

- In what cohort of young people should the medication be used?

- If accepted, would the medication be initiated or continued in primary care by General Practitioners (GPs), where a shared care protocol would be developed to ensure the roles and responsibilities of the specialist, GP and patient were clear to ensure appropriate prescribing and monitoring.

Results Following this appraisal, a positive evaluation and subsequent approval from the National Health Service (NHS) resulted in submission of application to the Area Prescribing Committee (APC) covering 3 cities, UK. The APC consists of varied healthcare individuals including the original sponsor to assess the formulary application and reach an informed decision. Once successful the medication is colour coded:

- Green enables all prescribers in health care to prescribe without restriction.
- Amber defines that initiation is performed by specialists in secondary care setting and prescribing transferred to GPs when the medication has been stabilised.

- Red requir es initiation and maintenance only by the specialist.

Conclusion This pathway for the approval of medication to treat ADHD was designed in order to provide a consistent process and enable young people to receive evidence-based cost-effective treatment. This enables clinicians to have a transparent process across different health care settings.

Recommendation This approach can be transferred across regions in NHS (National health service) and would be useful to compare in future the processes in other regions/countries. An audit across the three cities and survey of clinicians about it would be useful to inform other practices.

\section{G631(P) AN AUDIT OF PAEDIATRIC NEURODISABILITY MODEL OF CARE IN IRELAND}

Ar Rasool. Paediatrics, Portiuncula University Hospital, Galway, Ireland

\subsection{6/archdischild-2020-rcpch.545}

Background It is estimated that $4 \%$ of children have complex on-going disability needs for health supports, with a further $10 \%$ having occasional needs for health intervention (HSE, 2009). A National Model of Care for Paediatric Healthcare Services in Ireland has been proposed by HSE for management of patients with neurodisability with an aim of providing best standard of care for children and young people with disability. However, there is paucity of data on the availability and ease of access of these available services; warranting this audit.

Aims

- To audit waiting period for accessing a facility for a patient with neurodisability

- To compare the waiting period in facilities inside the hospital vs in other hospital and in the community.

Methods A retrospective audit of referrals of patients with neurodisability in Paediatric department of Portiuncula University Hospital, Ballinasloe was done in July 2019. Referrals to medical and non-medical services including early intervention team were included in the study. Referrals for urgent transfer for any medical needs to a specialized setup were not included in the audit. The time at which referral was made was documented in 'weeks of life' of the corresponding patient. We audited the time at which referral was made and the time it got accepted for relevant action. Ease was access was ascertained average waiting time. We also audited the difference in time in intra-hospital vs inter-hospital referrals.

Results A total of 31 referrals were audited. Referral rejection percentage was $3 \%$. Three percent of referrals were still awaiting appropriate response. A total of 9 emergency transfers within 6 months of life were documented. Average waiting period was 10 weeks with Standard deviation of 14.8 weeks. Waiting period for intra-hospital referrals (services available at PUH) was 0.8 weeks compared to 20.66 weeks for inter-hospital (for services at Dublin, Galway-ophthalmology, ENT etc) referrals. Waiting period for services in community was 5.2 weeks for EIT, social worker etc. Access to in 